\title{
Lidil
}

Revue de linguistique et de didactique des langues

$64 \mid 2021$

Le passif dans la langue parlée

\section{Usage du passif dans un corpus oral en capverdien santiagais}

The Use of Passive Voice in Spoken Santiaguense Capeverdean: A Corpus-Based Approach

\section{Nicolas Quint et Eliane Vieira Semedo}

\section{CpenEdition}

\section{Journals}

Édition électronique

URL : https://journals.openedition.org/lidil/9999

DOI : $10.4000 /$ lidil. 9999

ISSN : $1960-6052$

Éditeur

UGA Éditions/Université Grenoble Alpes

Édition imprimée

ISBN : 978-2-37747-315-1

ISSN : $1146-6480$

\section{Référence électronique}

Nicolas Quint et Eliane Vieira Semedo, «Usage du passif dans un corpus oral en capverdien santiagais », Lidil [En ligne], 64 | 2021, mis en ligne le 01 novembre 2021, consulté le 25 novembre 2021. URL : http://journals.openedition.org/lidil/9999 ; DOI : https://doi.org/10.4000/lidil.9999

Ce document a été généré automatiquement le 25 novembre 2021.

(c) Lidil 


\title{
Usage du passif dans un corpus oral en capverdien santiagais
}

The Use of Passive Voice in Spoken Santiaguense Capeverdean: A Corpus-Based Approach

\author{
Nicolas Quint et Eliane Vieira Semedo
}

\section{NOTE DE L'AUTEUR}

Metadados em português

Título:

O uso da voz passiva em um corpus oral de cabo-verdiano santiaguense

Palavras-chaves:

cabo-verdiano, corpus, crioulos de base lexical portuguesa da África Ocidental (CPAO),

línguas românicas, português, voz passiva

\section{Introduction}

1 La langue capverdienne (LCV) est la langue maternelle de la grande majorité des habitants de la république du Cap-Vert en Afrique de l'Ouest, pays-archipel dont la langue officielle est le portugais.

2 Le capverdien santiagais (ST), parlé sur l'île de Santiago, possède deux voix verbales exprimées de façon synthétique ${ }^{1}$, à savoir l'actif et le passif. La voix active n'a pas de marque segmentale (morphème $ø$ ou zéro ${ }^{2}$ ), tandis que la voix passive est caractérisée par la désinence verbale '-[du] au présent et '-[de] au passé. Au niveau de l'usage (Baptista, 2002, p. 112-113 ; Creissels et coll., 2015, p. 62 ; Quint, 2000a, p. 234), la voix active dénote le fait que l'action est effectuée par le sujet (sujet = agent), et la voix passive est employée essentiellement dans deux types de situations : 
une action où le sujet est exprimé et a un rôle sémantique de patient (ex. 01) :

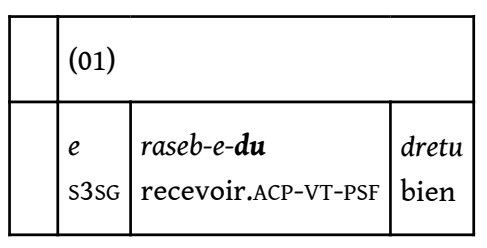

'Il a été bien reçu.'

(Quint, 2000a, p. 234)

2. une action dont le sujet est impersonnel (collectif ou indéfini) (ex. 02) :

\begin{tabular}{|l|l|l|l|}
\hline \multicolumn{3}{|l|}{ (02) } \\
\hline & $\begin{array}{l}|c| \\
\text { fas-e-du } \\
\text { faire.ACP-VT-PSF }\end{array}$ & $\begin{array}{l}\text { fésta } \\
\text { fête }\end{array}$ & $\begin{array}{l}\text { bedju } \\
\text { extraordinaire (lit. 'vieux') }\end{array}$ \\
\hline
\end{tabular}

'On a fait une grande fête, les gens ont fait une grande fête.'

(Quint, 2000a, p. 234)

3 La présente étude a pour objectif de revisiter la question du passif en capverdien en se fondant sur le dépouillement exhaustif d'un corpus oral produit par des locuteurs basilectaux ${ }^{3}$ du santiagais rural. Dans une première partie, nous présenterons la langue capverdienne et la méthodologie utilisée pour la collecte des données. Dans une deuxième partie, nous donnerons des informations générales sur la morphologie du passif en capverdien santiagais. Puis, dans une troisième partie, nous étudierons l'usage du passif attesté dans notre corpus en insistant notamment sur (i) les fréquences relatives des constructions passive et active (Givón, 2001); (ii) le rapport entre la marque de passif et les marques d'aspect et de temps (les deux principaux autres types de morphèmes verbaux en capverdien, cf. Quint (2000a)) ainsi que de polarité ; (iii) la syntaxe du passif (actants impliqués et degré de transitivité des verbes attestés dans des constructions passives); (iv) le profil lexical des verbes passifs. Nous vérifierons notamment si les phénomènes (i) à (iv) observés en corpus confirment ou infirment les observations déjà faites par Quint (2000a) et d'autres auteurs sur la voix passive en capverdien.

\section{Données linguistiques et méthodologiques}

\subsection{La langue capverdienne : présentation et choix de la variété étudiée}

4 Aujourd'hui, la LCV est la langue maternelle d'environ un million de personnes vivant dans la république du Cap-Vert ou provenant de ce pays. En république du Cap-Vert 
(environ 580000 habitants), la proportion de locuteurs natifs du capverdien est probablement supérieure à $95 \%^{4}$. En pratique, le capverdien est quasiment la seule langue parlée dans la vie courante, tant en milieu urbain que rural (où la proportion de locuteurs est souvent de $100 \%$ ).

La LCV est un créole à base lexicale portugaise dont la majorité du lexique est issue du portugais classique (1450-1650) tandis que divers éléments de la langue, en particulier au niveau de la morphosyntaxe, proviennent de plusieurs langues africaines de substrat, essentiellement le mandinka, le wolof et le temné (Lang, 2006, p. 53-62 ; 2009a ; 2009b, p. 61-80 ; Quint, 2000b, p. 32 ; 2006, p. 88 ; Quint \& Moreira, 2019, p. 120). La LCV n'étant pas enseignée dans le système scolaire, la production écrite est limitée (la principale langue écrite au Cap-Vert est le portugais) et on peut dire que la LCV reste fondamentalement une langue orale.

6 La variation interne à la LCV s'organise autour de deux axes principaux (Quint, 2000a, p. $9-10 ; 2000$ b, p. 95-97) :

1. variation diatopique: les neuf îles habitées de l'archipel du Cap-Vert ont chacune une variété propre de la LCV, souvent aisément identifiable par les locuteurs des autres îles. Ces variétés insulaires se regroupent en deux grands ensembles: (a) les parlers du Sud, pratiqués dans les Îles-Sous-le-Vent (en portugais: Sotavento), incluant les îles de Brava, Fogo, Maio et Santiago; (b) les parlers du Nord, pratiqués dans les îles-au-Vent (en portugais: Barlavento), incluant les îles de Boa Vista, Saint-Antoine (Sant'Antão), SaintNicolas (São Nicolau), Saint-Vincent (São Vicente) et Sal ;

2. l'influence du portugais : le portugais, langue officielle de la république du Cap-Vert, langue d'enseignement dans le système scolaire et dans l'administration, largement utilisé dans les médias audiovisuels et dominant de façon quasi exclusive la sphère de l'écrit, est une langue de prestige dans le système social capverdien. Le degré d'influence du portugais permet de classer les variétés capverdiennes d'une même île en deux pôles : (a) les parlers acrolectaux, plus influencés par le portugais et typiquement pratiqués par des personnes urbaines et ayant un niveau d'étude élevé; (b) les parlers basilectaux, plus éloignés de la langue lexificatrice (le portugais) et typiquement pratiqués par des personnes rurales et ayant peu ou pas fait d'études 5 .

7 La présente étude porte sur une variété diatopique particulière: le capverdien santiagais, une variété capverdienne du Sud, parlée traditionnellement sur l'île de Santiago et pratiquée en tant que langue première ou seconde par au moins $55 \%$ des Capverdiens résidant au Cap-Vert ${ }^{6}$. Au sein de Santiago, nous nous sommes intéressés à des variétés basilectales, pratiquées par des personnes âgées vivant en milieu rural: en effet, étant donné que celles-ci présentent moins d'influences du portugais moderne, dans une perspective contrastive, ces variétés permettent de mieux comprendre ce qui caractérise la grammaire de la langue capverdienne par rapport à celle du portugais.

\subsection{Recueil et constitution du corpus utilisé}

Le corpus sur lequel se fonde la présente étude contient 12 contes traditionnels en capverdien santiagais basilectal, représentant un total de $1 \mathrm{~h} 39 \mathrm{~min}$ de texte oral (y compris 4057 verbes fléchis). Ces contes ont été recueillis in situ par Nicolas Quint et Eliane Vieira Semedo en 2004, 2005 et 2015, dans différents villages de l'arrière-pays santiagais auprès de personnes encore habituées à raconter le répertoire traditionnel oral capverdien. Les enregistrements numériques collectés ont ensuite été 
systématiquement transcrits et analysés morphologiquement et syntaxiquement sous ELAN $(2020)^{7}$, dans le cadre d'un projet doctoral portant sur la phrase complexe en LCV (Vieira Semedo, en préparation).

Le genre choisi, celui de la littérature orale, est particulier et il n'a pas la prétention de représenter l'ensemble des registres disponibles en capverdien oral. Le corpus ici utilisé a néanmoins plusieurs avantages : d'un côté, il illustre un niveau conservateur de langue, manié par des conteuses et des conteurs habitués à produire des performances orales reconnues par la communauté. D'un autre côté, le genre du conte offre aussi une certaine liberté au narrateur (en fonction de son auditoire, de son expérience ou de son humeur) et il reflète donc plus fidèlement la langue orale du quotidien (nombre de passages des contes sont constitués de dialogues entre les personnages) que des types littéraires plus contraignants au niveau de la forme, tels que la chanson ou la poésie.

\section{Morphologie du passif en capverdien santiagais}

\subsection{Nature et origine des marques de passif en santiagais}

Comme signalé dans l'introduction, la marque du passif est '-[du] au présent et '-[de] au passé en capverdien santiagais.

11 Historiquement, la forme '-[du] provient du participe passé portugais (Lopes da Silva, 1957, p. 146 ; Quint, 2000a, p. 234 ; 2008, p. 132-33). En portugais, le passif est produit, comme en français, au moyen d'une périphrase verbale et répond à la formule ser 'être' + participe passé (ex.03a). En santiagais, comme dans les autres variétés capverdiennes du Sud, le verbe auxiliaire ser 'être' du portugais(PT) a été systématiquement élidé dans les constructions passives et, de ce fait, l'ancien participe portugais est devenu une forme finie dans le système morphologique de la LCV (ex. 03b).

(03a)

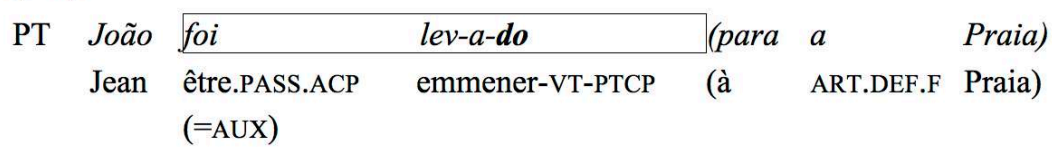

'Jean a été emmené à Praia'.

(03b)

\begin{tabular}{|c|c|c|}
\hline T ['dzõ & le'badu & 'praje] \\
\hline Djon & leb-á-du & (Práia) \\
\hline Jean & emmener.ACP-VT-PSF & (Praia) \\
\hline
\end{tabular}

En synchronie, la marque santiagaise de passif '-[du] contraste avec l'absence de marque, qui caractérise la voix active (ex. 04).

(04)

\begin{tabular}{|c|c|c|c|}
\hline $\mathrm{T} \quad\left[\mathrm{me} e^{\prime}\right.$ 'je & 'lebe & 'dzõ & 'praje] \\
\hline Mariâ & leb-a & Djon & (Práia) \\
\hline Marie & emmener.ACP-VT & Jean & (Praia) \\
\hline
\end{tabular}


Quant à la forme du passif passé '-[de], elle provient historiquement de la fusion d'une suite de deux morphèmes '-[du] (passif présent) + '-[be] (passé) > '-[dube] > '-[de]. La forme bisyllabique de passif passé '-[dube] est encore attestée dans la première grammaire capverdienne connue (Paula Brito, 1967 [1885], p. 376-380 ; Quint, 2008, p. 133-134) : elle semble aujourd'hui avoir été complètement remplacée par '-[de]. En pratique l'usage de '-[de] (comme celui de '-[be] à l'actif: cf. Quint (2000a, p. 235; 2003, p. 137 ; 2010, p. 139)) a pour effet de transposer dans la sphère du passé la construction passive présente (ex. 05-06) .

\begin{tabular}{|c|c|c|c|c|c|}
\hline \multicolumn{6}{|l|}{ (05) } \\
\hline Djon & $t a$ & odj-á-du & tudu & diâ & la \\
\hline Jean & INACP & voir-VT-PSF & tout & jour & là-bas \\
\hline
\end{tabular}

‘On voit Jean quotidiennement dans cet endroit', lit. 'Jean est vu tous les jours là-bas'.

\begin{tabular}{|c|c|c|c|c|c|}
\hline \multicolumn{6}{|l|}{ (06) } \\
\hline Djon & ta & odj-á-da & tudu & diâ & la \\
\hline Jean & INACP & voir-VT-PSF.PASS & tout & jour & là-bas \\
\hline
\end{tabular}

'On voyait Jean quotidiennement dans cet endroit', lit. 'Jean était vu tous les jours là-bas'.

\subsection{Propriétés combinatoires du passif santiagais}

D'un point de vue strictement morphologique, les marques de passif '-[du] et '-[de] sont suffixées à la forme clitique du verbe (tableau 1), comme les autres suffixes verbaux du capverdien (Quint, 2003, p. 232-233; 2004, p. $79 ; 2011$, p. 192 ; 2012, p. 162).

Tableau 1. - Morphologie du passif présent des verbes santiagais en fonction de la voyelle thématique (VT).

\begin{tabular}{|l|l|l|l|l|}
\hline VT & Forme libre & Forme clitique & Passif présent & Sens \\
\hline [a] & odja ['odze] & [o'dza] & odjádu [o'dzadu] & 'voir' \\
\hline [e] & djobi ['dzobi] & [dzo'be] & djobedu [dzo'bedu] & 'regarder' \\
\hline [i] & obi ['obi] & [o'bi] & obidu [o'bidu] & 'entendre' \\
\hline [o] & konpu ['kõpu] & [kõ'po] & konpodu [kõ'podu] & 'réparer' \\
\hline [u] & bonbu ['bõbu] & [bõ'bu] & bonbudu [bõ'budu] & 'porter sur le dos' \\
\hline
\end{tabular}




\begin{tabular}{|l|l|l|l|}
\hline$[\tilde{e}]$ & ben ['bẽ] & bendu ['bẽdu] & 'venir' \\
\hline
\end{tabular}

Comme le verbe capverdien n'accepte qu'un seul suffixe, l'objet d'un verbe au passif ne peut pas être un pronom clitique suffixé : les pronoms objets suivant un verbe passif sont donc toujours toniques (TON, ex. 08), alors que ceux suivant un verbe actif (non suffixé) sont clitiques (cL, ex. 07).

\begin{tabular}{|l|l|l|l|}
\hline \multicolumn{3}{|l|}{$(07)$} \\
\hline & {$[\mathrm{nu}$} & $\mathrm{ke}$ & o'd $3 \mathrm{el}]$ \\
$\mathrm{nu}$ & $k a$ & odj-â-l \\
& S1PL & $\mathrm{NEG}$ & voir.ACP-VT-03SG.CL \\
\hline & \multicolumn{2}{|l|}{ 'Nous ne l'avons pas vu.' } \\
\hline
\end{tabular}

\begin{tabular}{|l|l|l|l|}
\hline & \multicolumn{2}{|l|}{$(08)$} & \multicolumn{2}{|l|}{} \\
\hline & {$[\mathrm{nu}$} & $\mathrm{ke}$ & 'dadu-'el] \\
$n u$ & $k a$ & $d-a ́-d u-e l$ \\
& S1PL & NEG & donner.ACP-VT-PSF-03SG.TON \\
\hline & \multicolumn{2}{|l|}{ 'On ne nous l'a pas donné', lit. 'nous n'avons pas été donné lui'. } \\
\hline
\end{tabular}

16 Par ailleurs, le passif est compatible avec toutes les particules aspectuelles et modales $\mathrm{du}$ capverdien santiagais: (i) accompli : marque zéro (ex.01-04 et 07-08), (ii) inaccompli : marque ta (ex. 05-06), et (iii) progressif : marque sata (ex. 09), etc.

\begin{tabular}{|l|l|l|l|}
\hline & \multicolumn{2}{|l|}{$(09)$} \\
\hline & $\begin{array}{l}\text { N } \\
\text { S1SG }\end{array}$ & $\begin{array}{l}\text { sata } \\
\text { PROG }\end{array}$ & $\begin{array}{l}\text { sper-á-du } \\
\text { attendre-VT-PSF }\end{array}$ \\
\hline & 'On m'attend', lit. 'je suis en train d'être attendu'. \\
\hline
\end{tabular}

17 On notera enfin que le verbe ser 'être' ne semble pas être attesté au passif (on attendrait des formes passives $*[$ 'serdu] au présent et *['serde] au passé).

\subsection{La question du complément d'agent}

Contrairement aux langues romanes, le passif capverdien n'est normalement pas compatible avec un complément d'agent (Quint, 2000a, p. $234 ; 2003$, p. 137 et $255 ; 2010$, p. 139 et 257) ${ }^{9}$. Lorsque l'identité de l'agent doit être dévoilée en discours, le capverdien recourt à une phrase explicative à la voix active, comme on peut le voir en (ex. 10) qui fournit un équivalent de la phrase française 'Jean a été tué par son ami'. 


\begin{tabular}{|l|l|l|}
\hline \multicolumn{2}{|l|}{ (10a) } \\
\hline & $\begin{array}{l}\text { Djon } \\
\text { S3SG }\end{array}$ & $\begin{array}{l}\text { mat-á-du } \\
\text { tuer.ACP-VT-PSF }\end{array}$ \\
\hline & 'Jean a été tué.' \\
\hline
\end{tabular}

\begin{tabular}{|c|c|c|c|}
\hline \multicolumn{4}{|l|}{$(10 b)$} \\
\hline $\begin{array}{l}\text { Si } \\
\text { POSS3SG }\end{array}$ & $\begin{array}{l}\text { amigu } \\
\text { ami }\end{array}$ & $\begin{array}{l}k i \\
\text { REL.FOC.S }\end{array}$ & $\begin{array}{l}\text { mat- } \hat{a}-l \\
\text { tuer.ACP-VT-O3SG }\end{array}$ \\
\hline \multicolumn{4}{|c|}{ 'C'est son ami qui l'a tué.' } \\
\hline
\end{tabular}

19 Notre corpus confirme les travaux précédents pour le santiagais basilectal, puisqu'aucun complément d'agent n'a effectivement été trouvé dans les constructions passives relevées ${ }^{10}$.

\section{Analyse du corpus}

\subsection{Fréquences relatives des constructions actives et passives}

20 Tout d'abord, on doit noter que les constructions passives sont rares en capverdien oral $^{11}$, au moins au sein de notre corpus : sur un total de 4057 verbes fléchis dans le corpus, seuls 65 verbes le sont au passif (soit moins de $2 \%$ du total). Autre indice de cette rareté, deux des 12 contes dépouillés ne comportent aucune forme de passif et trois autres contes ne comportent qu'une forme de passif chacun. Cependant, quoique peu fréquentes, les formes passives apparaissent tout de même dans 10 contes sur 12, preuve que leur usage est relativement solide et régulier. Les constructions passives constituent donc clairement des procédés syntaxiques marqués (Keenan \& Dryer, 2007, p. 325-326), mais pas exceptionnels.

21 Si l'on s'intéresse aux types de propositions dans lesquels apparaissent les formes passives (tableau 2), on constate que leur répartition correspond dans l'ensemble à celle observée dans l'ensemble du corpus. Deux écarts apparaissent néanmoins :

1. les formes passives semblent apparaitre plus fréquemment dans des propositions complétives ( $42 \%$ contre $32 \%$ pour l'ensemble du corpus, ex. 11-13) :

\begin{tabular}{|l|l|l|l|}
\hline \multicolumn{2}{|l|}{$(11)$} & \multicolumn{2}{|l|}{} \\
\hline & $N$ & $k a$ & $f l-a-u$ \\
S1SG & NEG & dire.ACP-VT-O2SG \\
\hline
\end{tabular}




\begin{tabular}{|l|l|l|l|l|l|}
\hline $\begin{array}{l}\text { [ma } \\
\text { COMP } \\
\text { [COMPLÉTIVE }\end{array}$ & DEIC.PRCH & $k a$ & NEG & INACP & $\begin{array}{c}\text {-en-du] } \\
\text { venir-VT-PSF } \\
]\end{array}$ \\
\hline
\end{tabular}

'Ne t'ai-je pas dit qu'il ne faut pas venir ici ?', lit. 'qu'ici on ne vient pas'.

\begin{tabular}{|c|c|c|c|c|}
\hline (12) & & & & \\
\hline $\begin{array}{l}e \\
\text { S3SG } \\
\text { ÉNONCÉ } 1\end{array}$ & $\begin{array}{l}\text { txig- } a \\
\text { arriver.ACP-VT }\end{array}$ & $\begin{array}{l}n a \\
\text { à }\end{array}$ & $\begin{array}{l}\text { un } \\
\text { INDF }\end{array}$ & $\begin{array}{l}\text { sidádi } \\
\text { ville }\end{array}$ \\
\hline
\end{tabular}

\begin{tabular}{|l|l|l|l|l|l|l|l|}
\hline $\begin{array}{l}n a \\
\text { à } \\
\text { ÉNONCÉ 2 }\end{array}$ & $\begin{array}{l}\text { kel } \\
\text { DEM.ELGN }\end{array}$ & $\begin{array}{l}\text { sidádi } \\
\text { ville }\end{array}$ & $\begin{array}{l}\text { S S3SG } \\
\text { trouver.ACP-VT }\end{array}$ & $\begin{array}{l}\text { áta } a \\
\text { INACP }\end{array}$ & $\begin{array}{l}\text { djug-á-du] } \\
\text { jouer-VT-PSF }\end{array}$ \\
\hline PRINCIPALE & & [COMPLÉTIVE] \\
\hline & $\begin{array}{l}\text { 'Il arriva dans une ville [et] dans cette ville, il trouva des gens en train de jouer', lit. 'il trouva } \\
\text { [c'est] en train d'être joué'. }\end{array}$ \\
\hline
\end{tabular}

\begin{tabular}{|l|l|l|l|l|l|l|}
\hline \multicolumn{6}{|l|}{ (13) } \\
\hline & $\begin{array}{l}\text { Frakasi } \\
\text { Frakasi (NPR) } \\
\text { PRINCIPALE }\end{array}$ & $\begin{array}{l}\text { sub-i } \\
\text { monter.ACP-VT }\end{array}$ & $\begin{array}{l}\text { na } \\
\text { sur }\end{array}$ & $\begin{array}{l}\text { monti } \\
\text { montagne }\end{array}$ & $\begin{array}{l}\text { si } \\
\text { ainsi }\end{array}$ & $\begin{array}{l}f l-a: \\
\text { dire.ACP-VT }\end{array}$ \\
\hline
\end{tabular}

\begin{tabular}{|l|l|l|l|c|}
\hline $\begin{array}{l}\text { 'Argen! } \\
\text { quelqu'un } \\
\text { [COMPLÉTIVE }\end{array}$ & $\begin{array}{l}\text { Bu } \\
\text { S2SG }\end{array}$ & $\begin{array}{l}\text { INACP } \\
\text { INAom-á-du } !^{\prime 12}\end{array}$ & $\begin{array}{c}\text { appeler-VT-PSF } \\
]\end{array}$ \\
\hline
\end{tabular}

‘Frakasi monta donc sur une montagne et il s'écria (lit. 'dit') : ‘Eh toi l'inconnu ! On t’appelle !”, lit. 'quelqu'un! Tu es appelé !'

2. les formes passives semblent particulièrement rares dans les propositions adverbiales ( $2 \%$ contre $10 \%$ pour l'ensemble du corpus, soit un rapport de 1 à $5^{13}$ ). Il est cependant possible que cet écart soit lié à la taille de l'échantillon (une seule forme passive est attestée dans une proposition adverbiale). 
Tableau 2. - Fréquences relatives des constructions passives en fonction du type de proposition attesté.

\begin{tabular}{|c|c|c|c|c|}
\hline & \multicolumn{2}{|c|}{ Constructions passives } & \multicolumn{2}{|c|}{ Ensemble du corpus } \\
\hline Propositions & Nombre & $\%$ & Nombre & $\%$ \\
\hline Coordonnées & 34 & $53 \%$ & 2016 & $52 \%$ \\
\hline Complétives & 27 & $42 \%$ & 1239 & $32 \%$ \\
\hline Relatives & 2 & $3 \%$ & 220 & $6 \%$ \\
\hline Adverbiales & 1 & $2 \%$ & 365 & $10 \%$ \\
\hline Insubordonnées ${ }^{14}$ & 0 & $0 \%$ & 12 & $0 \%$ \\
\hline Total & $64^{15}$ & $100,0 \%$ & $3852^{16}$ & $100,0 \%$ \\
\hline
\end{tabular}

\subsection{Passif, temps, aspect et polarité}

\subsubsection{Temps}

Comme on peut le voir dans le tableau 3, le temps passé (marqué par le suffixe '-[be] à l'actif et par le suffixe '-[de] au passif) a une fréquence très basse dans notre corpus ( $1 \%$ à l'actif, $5 \%$ au passif), où le présent ${ }^{17}$ (non marqué à l'actif et marqué par le suffixe '-[du] au passif) domine très fortement au niveau statistique. Ceci est en accord avec des tendances générales de la morphologie capverdienne, où les formes les moins marquées ${ }^{18}$ sont régulièrement privilégiées (Lang, en préparation, p. 147-149; Quint, 2000a, p. 343-346 ; 2003 ; Pereira, 1992 ; Vieira Semedo, en préparation).

En revanche, il ne semble pas y avoir de différence significative d'emploi du passé dans les énoncés au passif par rapport à l'ensemble du corpus. Le paramètre TEMPS n'a donc pas d'effet sur l'emploi du passif.

Tableau 3. - Fréquences relatives des formes passées au passif et dans l'ensemble du corpus.

\begin{tabular}{|l|l|l|l|l|}
\hline & \multicolumn{2}{|l|}{ Constructions passives } & \multicolumn{2}{l|}{ Ensemble du corpus } \\
\hline Temps & Nombre & $\%$ & Nombre & $\%$ \\
\hline Présent & 62 & $95 \%$ & 4015 & $99 \%$ \\
\hline Passé & 3 & $5 \%$ & 42 & $1 \%$ \\
\hline Total & 65 & $100,0 \%$ & 4057 & $100,0 \%$ \\
\hline
\end{tabular}




\subsubsection{Aspect}

Le passif semble interagir significativement avec l'aspect verbal en capverdien santiagais ${ }^{19}$ (tableau 4) : en effet, l'inaccompli est visiblement plus fréquent dans les constructions passives que dans l'ensemble du corpus (34\% contre $13 \%$, soit un rapport de presque 3 contre 1 ).

Tableau 4. - Fréquences relatives des marques aspectuelles au passif et dans l'ensemble du corpus.

\begin{tabular}{|l|l|l|l|l|l|}
\hline & & \multicolumn{2}{|l|}{ Constructions passives } & \multicolumn{2}{l|}{ Ensemble du corpus } \\
\hline Aspect & Marque & Nombre & $\%$ & Nombre $^{20}$ & $\%$ \\
\hline Accompli & zéro (ø) & 42 & $64 \%$ & 3163 & $85 \%$ \\
\hline Inaccompli & ta [te] & 22 & $34 \%$ & 499 & $13 \%$ \\
\hline Progressif & $\begin{array}{l}\text { sata áta } \\
{[\text { sete 'ate] }}\end{array}$ & 1 & $2 \%$ & 63 & $2 \%$ \\
\hline Total & & 65 & $100,0 \%$ & 3725 & $100,0 \%$ \\
\hline
\end{tabular}

Les 22 constructions passives inaccomplies du corpus illustrent les trois valeurs reconnues par ailleurs pour la marque ta en capverdien (Quint, 2000a, p. 236-238; 2003, p. $244 ; 2005$, p. 43 ; 2010, p. 246-247), à savoir (i) habituel : 13 occurrences (ex. 11, 18-19); (ii) progressif (valeur proche de sata): 7 occurrences (ex. 12-13); (iii) prospectif : 2 occurrences (ex. 14).

\begin{tabular}{|l|l|l|l|l|}
\hline & \multicolumn{4}{|l|}{$(14)$} \\
\hline & $\begin{array}{l}\text { (...) gósi } \\
\text { maintenant }\end{array}$ & $\begin{array}{l}n u \\
\text { S1PL }\end{array}$ & $\begin{array}{l}\text { ta } \\
\text { INACP }\end{array}$ & $\begin{array}{l}\text { peg-á-du } \\
\text { attraper-VT-PSF }\end{array}$ \\
\hline & '[Il lui dit :] cette fois on va nous attraper', lit. 'maintenant, nous serons attrapés'. \\
\hline
\end{tabular}

Il n'a pas été possible de comparer ces valeurs de l'inaccompli en contexte passif avec le reste du corpus. En effet, lesdites valeurs, qui requièrent un examen au cas par cas de chaque énoncé en contexte, n'ont pas été prises en compte dans le processus d'annotation général.

\subsubsection{Polarité}

27 En termes quantitatifs (tableau 5), la polarité ne semble avoir aucun effet sur le passif puisque la proportion de formes négatives $(8 \%)$ est exactement la même que dans l'ensemble du corpus ${ }^{21}$. 
Tableau 5. - Fréquences relatives des formes négatives au passif et dans l'ensemble du corpus.

\begin{tabular}{|l|l|l|l|l|}
\hline & \multicolumn{2}{|l|}{ Constructions passives } & \multicolumn{2}{l|}{ Ensemble du corpus 22} \\
\hline Polarité & Nombre & $\%$ & Nombre & $\%$ \\
\hline Positive & 60 & $92 \%$ & 3742 & $92 \%$ \\
\hline Négative & 5 & $8 \%$ & 315 & $8 \%$ \\
\hline Total & 65 & $100,0 \%$ & 4057 & $100,0 \%$ \\
\hline
\end{tabular}

D'un point de vue qualitatif, on observe toutefois que quatre des cinq passifs négatifs attestés proviennent du même conte et contiennent tous la formule (ex. 15), répétée pour les besoins expressifs de la narration:

\begin{tabular}{|l|l|l|l|l|}
\hline & \multicolumn{3}{|l|}{$(15)$} \\
\hline & $\begin{array}{l}\text { li } \\
\text { DEIC.PRCH }\end{array}$ & $\begin{array}{l}k a \\
\text { NEG }\end{array}$ & $\begin{array}{l}\text { ta } \\
\text { INACP }\end{array}$ & $\begin{array}{l}\text { b-en-du } \\
\text { venir-VT-PSF }\end{array}$ \\
\hline & \multicolumn{2}{|l}{ 'Il ne faut pas venir ici', lit. 'ici, [il] n'est pas venu, ici [on] ne vient pas'. } \\
\hline
\end{tabular}

Si l'on considère que les quatre occurrences de li ka ta bendu ne constituent qu'un seul et même phénomène, on n'a en fait que deux cas distincts de passifs négatifs dans le corpus (ex. 15 et 16).

\begin{tabular}{|l|l|l|l|l|l|l|}
\hline & \multicolumn{5}{|l|}{$(16)$} \\
\hline $\begin{array}{l}N \\
\text { S1SG }\end{array}$ & $\begin{array}{l}\text { ta } \\
\text { INACP }\end{array}$ & $\begin{array}{l}\text { d-a-u } \\
\text { donner-VT-O2SG }\end{array}$ & $\begin{array}{l}\text { la } \\
\text { DEIC.ELGN }\end{array}$ & $\begin{array}{l}n a \\
\text { dans }\end{array}$ & $\begin{array}{l}\text { peli } \\
\text { peau }\end{array}$ \\
\hline $\begin{array}{l}k e l \\
\text { DEM.ELGN }\end{array}$ & $\begin{array}{l}\text { otu } \\
\text { autre }\end{array}$ & $\begin{array}{l}k a \\
\text { NEG }\end{array}$ & $\begin{array}{l}\text { ta } \\
\text { INACP }\end{array}$ & $\begin{array}{l}f l-a ́-d u \\
\text { venir-VT-PSF }\end{array}$ & \\
\hline & $\begin{array}{l}\text { 'Si tu viens avec moi], je te toucherai la 'peau' (euphémisme pour les parties sexuelles), [bon] le } \\
\text { vrai mot on ne doit pas le dire', lit. 'cet autre [mot] n'est pas dit, cet autre [mot] ne se dit pas'. }\end{array}$ \\
\hline
\end{tabular}

30 Ces deux cas de passifs négatifs ont en commun le fait qu'ils sont l'un comme l'autre employés avec des verbes à l'aspect inaccompli. Cependant, la petite taille de l'échantillon (deux formes distinctes) ne permet pas de savoir si ce point commun atteste d'une tendance générale dans la langue.

\subsection{Passif, actants et valence verbale}

31 Au niveau des sujets des constructions passives (tableau 6), les deux principales catégories sont (i) les passifs à sujet exprimé (catégorie des passifs canoniques de 
Creissels (2006, p. 43-44) ou des 'basic passives' de Keenan \& Dryer (2007, p. 328-342)) (ex. 01, 17) et (ii) les passifs à sujet impersonnel (Creissels, 2006, p. 53-54; Keenan \& Dryer, 2007, p. 345-348) (ex.02, 18). Ces deux catégories ont des fréquences sensiblement équivalentes dans le corpus (respectivement $54 \%$ et $46 \%$ ). La plupart des sujets exprimés sont des pronoms personnels ou des syntagmes nominaux (en tout $19+11=30 / 35$ soit $86 \%$ des sujets exprimés). On notera aussi qu'une proportion significative des sujets exprimés n'apparait pas dans la proposition où se trouve le verbe passif, mais dans un autre élément du contexte (proposition principale ou contexte plus large): c'est le cas (tableau 6) de 5 des 19 pronoms personnels sujets (ex. 19) et de 6 des 11 syntagmes nominaux sujets (ex. 20) de ce même tableau.

Tableau 6. - Sujets des verbes passifs attestés dans le corpus.

\begin{tabular}{|l|l|l|l|}
\hline & & \multicolumn{2}{l|}{ Constructions passives } \\
\hline & & Nombre & $\%$ \\
\hline Sujet exprimé & 35 & $54 \%$ \\
\hline dont : & pronoms personnel & 19 & $29 \%$ \\
\hline & syntagme nominal & 11 & $17 \%$ \\
\hline & démonstratif & 2 & $3 \%$ \\
\hline & pronom relatif & 2 & $3 \%$ \\
\hline & indéfini & 1 & $2 \%$ \\
\hline & Total & $\mathbf{3 0}$ & $\mathbf{4 6} \%$ \\
\hline
\end{tabular}

\begin{tabular}{|l|l|l|l|l|l|l|}
\hline & \multicolumn{4}{|l|}{$(17)$} \\
\hline & $\begin{array}{l}\text { (..) ês } \\
\text { S3PL }\end{array}$ & $\begin{array}{l}\text { kiri-á-du } \\
\text { élever-VT-PSF }\end{array}$ & $\begin{array}{l}\text { déntu } \\
\text { àl'intérieur }\end{array}$ & $\begin{array}{l}\text { d’ } \\
\text { de }\end{array}$ & $\begin{array}{l}\text { un } \\
\text { INDF }\end{array}$ & $\begin{array}{l}\text { manjador } \\
\text { mangeoire }\end{array}$ \\
\hline & \multicolumn{4}{|l}{} \\
\hline
\end{tabular}

\begin{tabular}{|c|c|c|c|c|}
\hline \multicolumn{5}{|l|}{ (18) } \\
\hline kei & bés & góra & $t a$ & nbark-á-da \\
\hline DEM.ELGN & fois & de.fait & INACP & embarquer-VT-PSF.PASS \\
\hline
\end{tabular}




\begin{tabular}{|c|c|c|c|c|c|}
\hline & $p a$ & & & & \\
\hline NEG & par & REL.LOC & être.ACP & maintenant & \\
\hline \multicolumn{6}{|c|}{$\begin{array}{l}\text { 'Or, à cette époque, [quand] on embarquait, [ce n'était] pas à l'endroit où on le fait (lit. '[c']est') } \\
\text { de nos jours'. }\end{array}$} \\
\hline
\end{tabular}

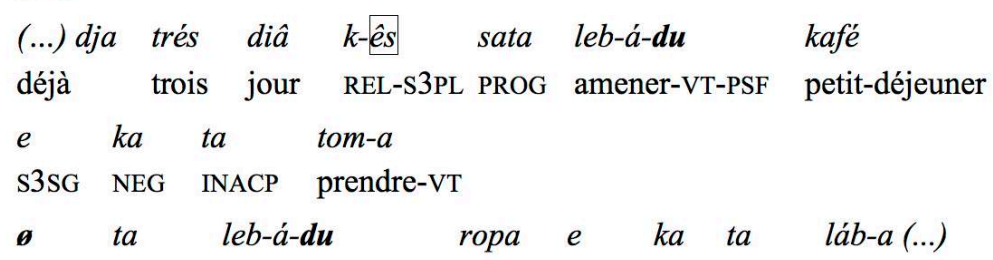

S3PL INACP amener-VT-PSF habits S3SG NEG INACP laver-VT

'Il y avait déjà trois jours qu'on leur amenait (lit. 'ils étaient amenés') le petitdéjeuner et que lui (lit. 'il') ne le prenait pas, qu'on leur amenait (lit. '[ils] étaient amenés') des vêtements et que lui (lit. 'il') ne se lavait pas (et donc n'utilisait pas les vêtements pour se changer après s'être lavé)'.

\begin{tabular}{|c|c|c|c|c|c|}
\hline $\begin{array}{l}\text { (...) } k i \\
\text { quel }\end{array}$ & $\begin{array}{ll}\text { diâ } & k i \\
\text { jour } & \text { REL.CC }\end{array}$ & $\begin{array}{l}b u \\
\text { s2sG }\end{array}$ & $\begin{array}{l}\text { odj-a } \\
\text { voir-vT }\end{array}$ & $\begin{array}{l}\text { sántxu } \\
\text { singe }\end{array}$ & $\begin{array}{l}\text { peg-á-du } \\
\text { attraper.ACP-VT-PSF }\end{array}$ \\
\hline ø torn- & \multicolumn{5}{|c|}{ larg-á-du } \\
\hline SN faire. & uveau. $A C P-V T$ & \multicolumn{4}{|c|}{ lâcher.ACP-VT-PSF } \\
\hline
\end{tabular}

'Quel jour a-t-on vu (lit. 'que tu as vu') qu'un singe soit attrapé puis [qu'il soit] relâché ?’

En ce qui concerne les compléments associés aux verbes passifs (tableau 7), presque la moitié de ces verbes (30 sur 65) n'ont pas de complément exprimé, généralement parce que la construction passive est intransitive (ex.17-18). La plupart des autres compléments sont des syntagmes nominaux (objets exprimés : 19 occurrences (ex. 19)) ou des propositions complétives (10 occurrences (ex. 21 et 25$)$ ), presque toutes $(9 / 10)$ dépendantes du seul verbe fla 'dire' (cf. tableau 7 ci-dessous).

Tableau 7. - Compléments des verbes passifs attestés dans le corpus.

\begin{tabular}{|l|l|l|}
\hline & \multicolumn{2}{|l|}{ Constructions passives } \\
\hline Type de complément & Nombre & $\%$ \\
\hline$\varnothing$ (zéro) & 30 & $46 \%$ \\
\hline Syntagme nominal & 19 & $29 \%$ \\
\hline Proposition complétive & 10 & $15 \%$ \\
\hline Adverbe & 4 & $6 \%$ \\
\hline Syntagme prépositionnel & 1 & $2 \%$ \\
\hline
\end{tabular}




\begin{tabular}{|l|l|l|}
\hline Pronom interrogatif & 1 & $2 \%$ \\
\hline Total & 65 & $100,0 \%$ \\
\hline
\end{tabular}

\begin{tabular}{|c|c|c|c|c|c|c|c|}
\hline \multicolumn{8}{|l|}{ (21) } \\
\hline kei & diâ & $f l-a ́-d u$ & {$[m a$} & la & fin & $d i$ & mundu \\
\hline DEM.ELGN & jour & dire.ACP-VT-PSF & COMP & DEIC.ELGN & fin & de & monde \\
\hline PRINCIPALE & & & [COMPLÉTIVE & & & & \\
\hline
\end{tabular}

\begin{tabular}{|l|l|l|l|l|}
\hline $\begin{array}{l}\text { ma } \\
\text { COMP }\end{array}$ & $\begin{array}{l}\text { t-en } \\
\text { y.avoir.ACP-VT } \\
\text { COMPLÉTIVE (suite) }\end{array}$ & $\begin{array}{l}\text { INDF } \\
\text { INmi } \\
\text { homme }\end{array}$ \\
\hline
\end{tabular}

\begin{tabular}{|l|l|l|l|l|l|}
\hline$k i$ & $\begin{array}{l}\text { t-en } \\
\text { REL.S }\end{array}$ & $\begin{array}{l}\text { trés } \\
\text { avoir.ACP-VT } \\
\text { COMPLÉTIVE (suite) }\end{array}$ & trois & $\begin{array}{l}\text { fidju } \\
\text { enfant }\end{array}$ & $\begin{array}{c}\text { fémia] } \\
\text { femelle } \\
]\end{array}$ \\
\hline
\end{tabular}

'Un beau jour (lit. 'ce jour'), quelqu'un dit (lit. 'il fut dit') que, tout au bout du monde, il y avait un homme qui avait trois filles'.

Nous avons aussi vérifié les constructions lexicales (attendues à la voix active) pour les verbes attestés au passif dans les contextes où ils apparaissent (tableau 8) :

1. la majorité de ces verbes sont transitifs lorsqu'ils sont employés à la voix active ;

2. les verbes lexicalement intransitifs sont exclusivement attestés dans des constructions passives impersonnelles, ce qui semble cohérent puisqu'un verbe intransitif ne permet pas la promotion d'un objet au rôle de patient ;

3. quant aux verbes ditransitifs, l'examen du corpus a révélé un trait syntaxique à notre connaissance non encore décrit pour le capverdien : dans une construction lexicalement ditransitive, seul l'objet récipiendaire RCP (à l'exclusion du patient PA) peut être promu au rang de sujet à la voix passive (ex. 22-23).

\begin{tabular}{|l|l|l|l|l|}
\hline \multicolumn{4}{|l|}{$\begin{array}{l}\text { (22a) } \\
\text { construction lexicale sous-jacente : }\end{array}$} \\
\hline $\begin{array}{l}\text { algen } \\
\text { quelqu'un } \\
\text { S }\end{array}$ & $\begin{array}{l}\text { leb- } \hat{a} \\
\text { amener.ACP-VT } \\
\text { V }\end{array}$ & $\begin{array}{l}-l \\
\text {-03SG } \\
\text { O1 (RCP) }\end{array}$ & $\begin{array}{l}\text { águ } \\
\text { eau } \\
\text { O2 (PA) }\end{array}$ \\
\hline \multicolumn{4}{|l}{} \\
'Quelqu'un lui a amené de l'eau'. \\
\hline
\end{tabular}

(22b)

construction passive attestée dans le corpus : 


\begin{tabular}{|l|l|l|l|}
\hline $\begin{array}{l}\boldsymbol{e} \\
\text { S3SG }\end{array}$ & $\begin{array}{l}\text { leb-á-du } \\
\text { amener.ACP-VT-PSF }\end{array}$ & $\begin{array}{l}\text { égu } \\
\text { eau }\end{array}$ \\
\hline
\end{tabular}

'On lui a amené de l'eau' (lit. 'il a été amené de l'eau') : c'est le récipiendaire (pronom de $3^{\mathrm{e}}$ personne du singulier avec un rôle sémantique de BÉNÉFICIAIRE) qui est promu au rang d'objet.

\begin{tabular}{|l|l|l|l|}
\hline \multicolumn{4}{|l|}{$\begin{array}{l}\text { (23a) } \\
\text { construction lexicale sous-jacente : }\end{array}$} \\
\hline $\begin{array}{l}\text { algen } \\
\text { quelqu'un } \\
\text { S }\end{array}$ & $\begin{array}{l}\text { bend- } \hat{e} \\
\text { vendre.ACP-VT } \\
\text { V }\end{array}$ & $\begin{array}{l}-l \\
\text {-03SG } \\
\text { O1 (RCP) }\end{array}$ & $\begin{array}{l}\text { kabálu } \\
\text { cheval } \\
\text { O2 (PA) }\end{array}$ \\
\hline
\end{tabular}

'Quelqu'un a vendu son cheval' (= pour le forcer à payer ses dettes), lit. 'quelqu'un lui a vendu [le] cheval'.

\begin{tabular}{|l|l|l|}
\hline & \multicolumn{3}{|l|}{$\begin{array}{l}(23 b) \\
\text { construction passive attestée dans le corpus : }\end{array}$} \\
\hline $\begin{array}{l}\boldsymbol{e} \\
\text { s3sG }\end{array}$ & $\begin{array}{l}\text { bend-e-du } \\
\text { vendre.ACP-VT-PSF }\end{array}$ & $\begin{array}{l}\text { kabálu } \\
\text { cheval }\end{array}$ \\
\hline
\end{tabular}

'On lui a vendu son cheval' (lit. 'il a été vendu le cheval') : c'est ici aussi le récipiendaire (pronom de $3^{\text {e }}$ personne du singulier avec un rôle sémantique de DÉTRIMENTAIRE) qui est promu au rang de sujet.

Nos vérifications auprès de locuteurs natifs ont montré que la promotion du patient au rang de sujet était effectivement systématiquement refusée pour les verbes ditransitifs.

Le corpus atteste par contre qu'un instrumental (cas de passif oblique, cf. Creissels (2006, p. 52)) peut aussi être promu au rang de sujet d'une passive (ex. 24) :

\begin{tabular}{|l|l|l|l|l|l|l|}
\hline & $\begin{array}{l}\text { (24a) } \\
\text { construction lexicale sous-jacente : }\end{array}$ \\
\hline $\begin{array}{l}\text { algen } \\
\text { quelqu'un } \\
\text { S }\end{array}$ & $\begin{array}{l}\text { fas-i } \\
\text { faire.ACP-VT } \\
\text { V }\end{array}$ & $\begin{array}{l}\text { djangáda } \\
\text { brancard } \\
\text { V }\end{array}$ & $\begin{array}{l}\text { nós } \\
\text { POSs1PL }\end{array}$ & $\begin{array}{l}\text { mai } \\
\text { mère }\end{array}$ & $\begin{array}{l}\boldsymbol{k} \boldsymbol{a v e c} \\
\text { INS }\end{array}$ & $\begin{array}{l}\text { karís } \\
\text { roseau }\end{array}$ \\
\hline & 'Quelqu'un a fabriqué le brancard mortuaire de notre mère avec de la canne de Provence.' \\
\hline
\end{tabular}


(24b)

construction passive attestée dans le corpus (structure focalisée) :

\begin{tabular}{|l|l|l|l|l|l|l|l|}
\hline $\begin{array}{l}\text { karís } \\
\text { roseau }\end{array}$ & $\begin{array}{l}k i \\
\text { REL.FOC.S }\end{array}$ & $\begin{array}{l}\text { fas-e-du } \\
\text { faire.ACP-VT-PSF }\end{array}$ & $\begin{array}{l}\text { djangáda } \\
\text { brancard }\end{array}$ & $\begin{array}{l}\text { nós } \\
\text { POSS1PL }\end{array}$ & $\begin{array}{l}\text { mai } \\
\text { mère }\end{array}$ & $\begin{array}{l}\boldsymbol{k u} \\
\mathbf{a v e c}\end{array}$ & $\begin{array}{l}\boldsymbol{e i} \\
\text { oBL3SG.TON }\end{array}$ \\
\hline & $\begin{array}{l}\text { 'C'est avec cette canne de Provence qu'on a fabriqué le brancard mortuaire de notre mère', lit. } \\
\text { 'c'est la canne de Provence qu'on a fait le brancard mortuaire de notre mère avec elle } \\
\text { (= la canne de Provence)' : l'instrumental (substantif karís) est ici promu au rang de sujet } \\
\text { focalisé et repris par un pronom de rappel (ei). }\end{array}$ \\
\hline
\end{tabular}

Tableau 8. - Valence lexicale (voix active) des verbes passifs attestés dans le corpus.

\begin{tabular}{|l|l|l|}
\hline & \multicolumn{2}{|l|}{ Constructions passives } \\
\hline Valence (voix active) & Nombre & $\%$ \\
\hline Intransitifs & 10 & $15 \%$ \\
\hline Transitifs & 41 & $63 \%$ \\
\hline Ditransitifs & 14 & $22 \%$ \\
\hline Total & 65 & $100,0 \%$ \\
\hline
\end{tabular}

\subsection{Profil lexical des verbes passifs}

Les 65 verbes passifs du corpus correspondent à 25 lexèmes verbaux, soit une fréquence moyenne de 2,6 $(=65 / 25)$ occurrences par lexème. La fréquence de ces lexèmes n'est cependant pas uniforme et elle permet de les classer en trois groupes statistiques (tableau 9) :

1. trois verbes passifs très fréquents (cinq occurrences ou plus + attestés dans au moins trois contes) : fla 'dire', fasi 'faire' et poi 'mettre'. Les fréquences cumulées de ces trois verbes représentent $38 \%(20+10+8)$ des occurrences de passif dans le corpus ;

2. quatre verbes passifs fréquents (deux occurrences ou plus + attestés dans deux contes) $: d a$ 'donner', lárga 'laisser', txoma 'appeler' et pega 'attraper';

3. verbes passifs peu fréquents (une ou plusieurs occurrences + attestés dans un seul conte) : 19 lexèmes verbaux. Certains de ces verbes apparaissent plusieurs fois dans le corpus, mais le fait que ces occurrences soient toutes liées à un seul conte est significatif: soit ces occurrences multiples sont dues à la reprise d'un motif lié à la structure du récit (cf. ci-dessus (ex. 15) le cas de bendu, passif du verbe ben 'venir', discuté en 3.2.3), soit elles sont dues à une hésitation du locuteur qui répète deux fois la forme en cherchant ses mots : dans notre corpus, seul obidu, passif du verbe obi 'entendre', correspond à ce cas de figure (ex. 25). Les occurrences multiples du passif dans un même conte ne peuvent donc pas être considérées comme l'indice d'une fréquence élevée d'usage du passif du verbe considéré dans la langue. 


\begin{tabular}{|l|l|l|l|l|l|}
\hline \multicolumn{2}{|l|}{$(25)$} \\
\hline $\begin{array}{l}\text { ob-i-du } \\
\text { entendre-VT-PSF } \\
\text { PRINCIPALE }\end{array}$ & $\begin{array}{l}\text { ob-i-du } \\
\text { entendre-VT-PSF }\end{array}$ & $\begin{array}{l}\text { fl-á-du: } \\
\text { dire.ACP-VT-PSF }\end{array}$ & $\begin{array}{l}\text { ['nhós } \\
\text { S2PL } \\
\text { [COMPLÉTIVE }\end{array}$ & $\begin{array}{l}\text { pára } \\
\text { arrêter.IMP }\end{array}$ & $\begin{array}{c}\text { pilon !'] } \\
\text { mortier } \\
\text { ] }\end{array}$ \\
\hline $\begin{array}{l}\text { 'Les gens ont entendu, ont entendu, [euh plutôt] ont dit : 'arrêtez de piler au mortier', lit. 'on a } \\
\text { entendu, on a entendu, on a dit : 'arrêtez le mortier !" }\end{array}$ \\
\hline
\end{tabular}

Tableau 9. - Fréquences relatives des lexèmes verbaux attestés dans les constructions passives et dans l'ensemble du corpus.

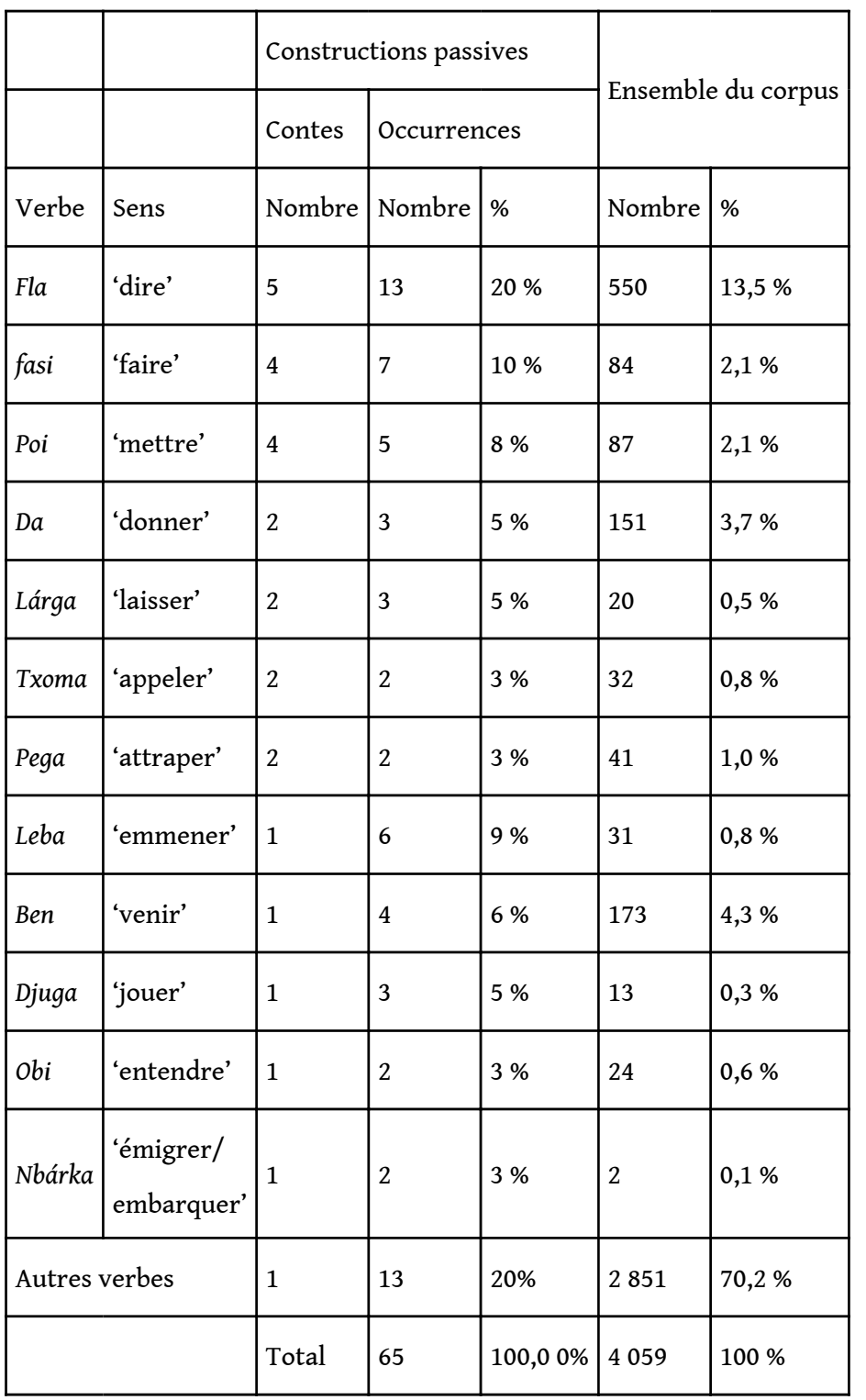

Les trois lexèmes verbaux les plus fréquents au passif sont également des verbes fréquents en général dans la langue, comme le montrent les données provenant de 
l'ensemble du corpus (tableau 9): fla 'dire' est probablement le verbe lexical (hors copules) le plus fréquent du corpus (13,5\% des occurrences de verbes) et probablement de la langue capverdienne.

Le profil syntaxique de ces trois lexèmes statistiquement dominants au passif révèle de nets contrastes (tableau 10):

- au passif, fla 'dire' est employé systématiquement (i) dans des propositions non subordonnées (coordonnées ou indépendantes), (ii) le plus souvent avec une valeur impersonnelle (10 occurrences/13) et (iii) son complément est généralement une proposition complétive (9 occurrences/13, ex. 21). En ce qui concerne (iii), ce résultat s'explique par le fait que fla est en capverdien le verbe déclaratif par excellence (Vieira Semedo et coll., 2018, p. 382) et que sa principale fonction est justement d'introduire des discours rapportés, généralement structurés sous la forme de complétives. Cette fonction d'introduction du discours rapporté explique aussi que fla soit surtout attesté dans des propositions coordonnées ou indépendantes (i). La prédominance d'un usage impersonnel du passif de fla (ii) laisse penser qu'il s'agit d'un recours oratoire permettant d'éviter de mentionner la source de l'information transmise par la complétive : de fait, le passif fládu de fla se traduit fréquemment en français par 'on dit';

- les données étant moins nombreuses, il est plus difficile de caractériser les profils syntaxiques des passifs de fasi 'faire' et poi 'mettre'. On note toutefois que, contrairement à fla, ils ne sont que (i) rarement employés avec un sujet impersonnel (1 occurrence chacun) et que (ii) leur objet n'est normalement pas une complétive ( 0 occurrence pour chaque verbe). Par ailleurs, (iii) fasi au passif tend à apparaitre préférentiellement dans des complétives, donc à dépendre d'un verbe introducteur.

Les caractéristiques sémantiques et syntaxiques de chaque lexème verbal interagissent donc à l'évidence avec le comportement de ce lexème au passif.

Tableau 10. - Profils syntaxiques des trois verbes les plus fréquemment attestés au passif dans le corpus.

\begin{tabular}{|l|l|l|l|l|}
\hline \multirow{4}{*}{} & & \multicolumn{3}{|c|}{ Verbe } \\
\hline \multirow{4}{*}{ Proposition } & & $\begin{array}{l}\text { fla } \\
\text { 'dire' }\end{array}$ & $\begin{array}{l}\text { fasi } \\
\text { 'faire' }\end{array}$ & $\begin{array}{l}\text { poi } \\
\text { 'mettre' }\end{array}$ \\
\cline { 2 - 5 } & Coord. / Indp. & 13 & 1 & 3 \\
\cline { 2 - 6 } & Complétive & 0 & 6 & 1 \\
\cline { 2 - 6 } & Adverbiale & 0 & 0 & 1 \\
\hline \multirow{5}{*}{ Sujet } & Total & $\mathbf{1 3}$ & $\mathbf{7}$ & 5 \\
\hline & Impersonnel & 10 & 1 & 1 \\
\cline { 2 - 6 } & SN & 2 & 2 & 2 \\
\cline { 2 - 6 } & Pronom & 1 & 4 & 2 \\
\hline
\end{tabular}




\begin{tabular}{|l|l|l|l|l|}
\hline \multirow{4}{*}{ Objet } & Complétive & 9 & 0 & 0 \\
\cline { 2 - 5 } & SN & 2 & 3 & 2 \\
\cline { 2 - 5 } & $\varnothing($ zéro) & 2 & 4 & 3 \\
\hline & Total & $\mathbf{1 3}$ & 7 & 5 \\
\hline
\end{tabular}

\section{Conclusion}

Cet article constitue la première étude consacrée au passif en capverdien qui soit fondée sur des données quantitatives issues du dépouillement systématique d'un corpus oral. Cette étude valide les résultats fournis par plusieurs publications et travaux de recherche précédents : ainsi, la description morphologique du passif et les deux grands types sémantiques identifiés (passif stricto sensu et impersonnel) sont confirmés par l'examen du corpus. En revanche, l'utilisation de corpus a permis de faire de remarquables progrès au niveau quantitatif et ce à de nombreux niveaux: (i) fréquence relative des formes passives; (ii) interaction entre aspect et passif (et absence d'interaction avec le temps et la polarité); (iii) types des sujets et des compléments associés de façon préférentielle à un verbe passif et valence lexicale des verbes fléchis au passif; (iv) profil lexical et syntaxique des verbes les plus fréquemment fléchis au passif, parmi lesquels fla 'dire', fasi 'faire' et poi 'mettre' semblent nettement dominer sur le plan statistique.

41 La présente recherche est bien entendu une étape et pas un point final. On peut espérer l'améliorer à l'avenir en suivant les axes suivants: (i) tout d'abord, de façon quantitative, en augmentant la taille du corpus, ce qui permettrait d'affiner certaines données (par exemple le profil des lexèmes les plus fréquemment fléchis au passif ou le comportement de formes rares telles que le passif passé); (ii) ensuite, sur le plan qualitatif: nous l'avons déjà dit (cf.1.2), le corpus actuel porte uniquement sur des contes traditionnels en créole basilectal. L'inclusion de nouveaux genres (dialogues, proverbes) et de nouveaux lectes (variétés urbaines, langue des jeunes...) modifierait forcément les résultats obtenus; (iii) la comparaison systématique des données présentées avec d'autres variétés de la langue capverdienne (parlers des autres îles), d'autres créoles apparentés (comme ceux de Casamance ou de Guinée-Bissao ou encore le papiamento, voir Jacobs (2011)) ou encore avec le portugais (langue lexificatrice du capverdien) pourrait à l'évidence offrir des perspectives fructueuses. Dans le même ordre d'idées, on pourrait aussi penser à des comparaisons entre registres de langue (tels que ceux évoqués ci-dessus en (ii)) et en particulier entre langue orale et langue écrite puisque, même si le portugais est la langue écrite dominante au Cap-Vert (cf. 1.1), il existe aujourd'hui un corpus conséquent de capverdien écrit; (iv) l'automatisation $d u$ traitement du corpus pourrait elle aussi être développée, en particulier au niveau de la transcription, ce qui permettrait justement d'obtenir de plus gros volumes textuels et des résultats plus fiables (cf. (i)).

Il reste donc certainement beaucoup à faire. En tout état de cause, la présente étude représente une avancée significative dans la connaissance et la description du passif verbal en capverdien contemporain. 


\section{BIBLIOGRAPHIE}

BAPTISTA, Marlyse. (2002). The Syntax of Cape Verdean Creole: The Sotavento Varieties. Benjamins.

BICKERTON, Derek. (1975). Dynamics of a Creole System. Cambridge University Press.

CREISSELS, Denis. (1991). Description des langues négro-africaines et théorie syntaxique. ELLUG.

CREISSELS, Denis. (2006). Syntaxe générale, une introduction typologique. La phrase (vol. 2). Hermès Science Publications / Lavoisier.

CReISSElS, Denis, BAO-Diop, Sokhna, BASSÈne, Alain-Christian, CISSÉ, Mame-Thierno, CoBBinAH, Alexander, DieYe, El-Hadji, NDAO, Dame, Nouguier-VoIsin, Sylvie, Quint, Nicolas, ReNAUdier, Marie, SALL, Adjaratou Oumar \& SEGERER, Guillaume. (2015). L'impersonnalité dans les langues de la région sénégambienne. Africana Linguistica, 21, 29-86.

ELAN [logiciel]. (2020). Nimègue : Max Planck Institute for Psycholinguistics, The Language Archive : <https://archive.mpi.nl/tla/elan>.

GIVóN, Talmy. (2001). Syntax: An Introduction (vol. 2). Benjamins.

HAMmA, Badreddine. (2017). Tentative de classification des « compléments d'agent » dans les phrases passives achevées et dans les énoncés longs à sens passif. Dans S. Gerolimich, M. de Gioia \& C. Martinot (dir.), Études de linguistique appliquée, 187(3), 311-324.

HAMmA, Badreddine. (2020). Pourquoi ne peut-on pas se passer de l'agent passif dans une vraie conversation ? Dans J. Chaker, L. Oueslati \& L. Hosni (dir.), Le dialogue et la conversation à la croisée des approches (p. 87-103). Latrach Édition.

HAMma, Badreddine, TARDIF, Amélie \& BADIN, Flora. (2017). Le passif à l'oral. Fiche du Projet FRACOV (Français contemporain vernaculaire) : <www.ortolang.fr/market/corpora/fracov? path $=\% 2 \mathrm{~F}>$.

JACOBS, Bart. (2011). The Origin and Originality of Passivization in Papiamentu. Journal of Portuguese Linguistics, 10(2), 31-56.

KEENAN, Edward \& DrYeR, Matthew. (2007). Passive in the World's Languages. Dans T. Shopen (dir.), Language, Typology and Syntactic Description: Clause Structure (vol. I, p. 325-361). Cambridge University Press.

LABATUT, Roger. (1976). La phrase peule et ses transformations (Thèse d'État). Université de Paris 3 Sorbonne Nouvelle.

LANG, Jürgen. (2006). L'influence des Wolof et du wolof sur la formation du créole santiagais. Dans J. Lang, H. John, J.-L. Rougé \& M. J. Soares (dir.), Cabo Verde: origens da sua sociedade e do seu crioulo (p. 53-74). Gunter Narr.

LANG, Jürgen. (2009a). A Wolof Trace in the Verbal System of the Portuguese Creole of Santiago Island (Cape Verde). Dans C. Lefebvre (dir.), Creoles, Their Substrates, and Language Typology (p. 61-80). Benjamins.

LANG, Jürgen. (2009b). Les langues des autres dans la créolisation. Théorie et exemplification par le créole d'empreinte wolof à l'île de Santiago du Cap-Vert. Gunter Narr.

LANG, Jürgen. (en préparation). Gramática do Crioula da ilha de Santiago (Cabo Verde). Manuscrit non publié. 
LOPES DA SILVA, Baltasar. (1957). o dialecto crioulo de Cabo Verde. Junta de Investigação do Ultramar, Centro de Estudos Políticos e Sociais.

MENDES, Mafalda, Quint, Nicolas, RAgAgelEs, Fátima \& SEMEDo, Aires. (2002). Dicionário prático português-caboverdiano. Verbalis.

PAULA BRITO, António DE. (1967). Apontamentos para a gramática do crioulo que se fala na ilha de Santiago de Cabo Verde. Dans J. Morais Barbosa (dir.), Estudos Linguísticos Crioulos (p. 329-404). Academia Internacional da Cultura Portuguesa.

PereirA, Dulce. (1992). O princípio da parcimónia em crioulo de Cabo Verde. Dans E. d'Andrade \& A. Kihm (dir.), Actas do Colóquio sobre «Crioulos de Base Lexical Portuguesa» (p. 141-151). Colibri.

QUINT, Nicolas. (2000a). Grammaire de la langue cap-verdienne : étude descriptive et compréhensive du créole afro-portugais des îles du Cap-Vert. L'Harmattan.

QUINT, Nicolas. (2000b). Le cap-verdien : origines et devenir d'une langue métisse. L'Harmattan.

QUINT, Nicolas. (2003). Parlons capverdien. L'Harmattan.

QUINT, Nicolas. (2004). Interações morfológicas entre verbo e objecto no crioulo da ilha de Santiago (República de Cabo Verde). Papia, 14, 71-83.

QUINT, Nicolas. (2005). Le créole capverdien de poche. Assimil.

QuinT, Nicolas. (2006). Un bref aperçu des racines africaines de la langue capverdienne. Dans J. Lang, H. John, J.-L. Rougé \& M. J. Soares (dir.), Cabo Verde: origens da sua sociedade e do seu crioulo (p. 75-90). Gunter Narr.

QUINT, Nicolas. (2008). Les apontamentos de António de Paula Brito (1887) ou la naissance d'une tradition grammaticale capverdienne autochtone. HEL - Histoire Épistémologie Langage, 1(30), 127-153.

QuiNT, Nicolas. (2010). Vamos falar caboverdiano. L'Harmattan.

QuINT, Nicolas. (2011). Le devenir du -/r/ de l'infinitif portugais en capverdien santiagais moderne. Dans A. Desporte \& G. Fabre (dir.), Aspects actuels de la linguistique ibéro-romane : actes du $\mathrm{XI}^{e}$ colloque international de linguistique ibéro-romane (p. 191-200). Lambert-Lucas.

QUINT, Nicolas. (2012). Les influences du portugais contemporain sur le système verbal $d u$ capverdien santiagais. Dans C. Chamoreau \& L. Goury (dir.), Changement linguistique et langues en contact : approches plurielles du domaine prédicatif (p. 155-178). CNRS Éditions.

QUINT, Nicolas \& MoReIRA TAVARES, Ana Karina. (2019). The Common African Lexical Core of the Upper Guinea Creoles and Its Historical Significance. Journal of Ibero-Romances Creoles, 9(1), 115-161. <www.acblpe.com/revista/volume-9-2019/the-common-african-lexical-core-of-theupper-guinea-creoles>.

VEIGA, Manuel. (2000). Le créole du Cap-Vert : étude grammaticale descriptive et constrative. Karthala.

VIEIRA SEMEDO, Eliane Cristina ARAÚJO. (en préparation). Frase complexa em cabo-verdiano: um estudo da integração entre cláusulas (Thèse de doctorat). Institut national des langues et civilisations orientales (INALCO), Paris / Université fédérale du Ceará (UFC), Fortaleza.

VIEIRA SEMEdo, Eliane Cristina ARAúJo, CoAn, Márluce \& QUINT, Nicolas. (2018). Orações completivas em cabo-verdiano. PAPIA - Revista Brasileira de Estudos do Contato Linguístico, 27(2), 367-383. <http:// revistas.fflch.usp.br/papia/article/view/2789>. 


\section{ANNEXES}

\section{Liste des abréviations}

\begin{tabular}{|c|c|c|c|}
\hline ACP & accompli & $0 / 0$ & objet \\
\hline ART & article & OBL & oblique \\
\hline FOC & focalisation & PA & patient \\
\hline $\mathrm{CL}$ & clitique & PASS & passé \\
\hline $\mathrm{CC}$ & complément circonstanciel & PL & pluriel \\
\hline сомР & conjonction complétive & POSS3SG & possessif $3^{\mathrm{e}}$ personne singulier \\
\hline DEF & défini & PRCH & proche \\
\hline DEIC & déictique & PROG & progressif \\
\hline DEM & démonstratif & PSF & passif \\
\hline $\mathrm{F}$ & féminin & PT & portugais \\
\hline ELGN & éloigné & РTCP & participe \\
\hline IMP & impératif & RCP & récipiendaire \\
\hline INACP & inaccompli & REL & relatif \\
\hline INDF & indéfini & $\mathrm{s} / \mathrm{s}$ & sujet \\
\hline INS & instrumental & SG & singulier \\
\hline LCV & Langue capverdienne & ST & santiagais \\
\hline LOC & locatif & TON & tonique \\
\hline NEG & négation & $\mathrm{v}$ & verbe \\
\hline NPR & nom propre & VT & voyelle thématique \\
\hline
\end{tabular}

\section{NOTES}

1. D'autres voix sont exprimées de façon analytique en capverdien : c'est notamment le cas du réfléchi et du réciproque (Quint, 2000a, p. 177-180 et 316 ; 2000b, p. 82-83;2003, p. 144 ; Veiga, 2000, p. 174-177).

2. Dans cet article, nous aurons régulièrement recours à la notion de morphème ou de marque zéro (ø). Nous ne prendrons cependant en compte l'existence de ø dans les transcriptions que 
lorsque son utilisation permet d'illustrer un contraste morphologique ou, de façon plus générale, a une utilité pour la démonstration.

3. Pour le sens donné au terme « basilectal » dans les études créoles, cf. note 5 ci-dessous.

4. Les principales minorités linguistiques sont constituées par des communautés d'origine étrangère: Africains continentaux (langues pratiquées traditionnellement en particulier en Sénégambie et en Guinée-Bissao - il y a probablement aujourd'hui plusieurs milliers de wolophones au Cap-Vert; on y rencontre également fréquemment des mandingophones et des locuteurs du peul - mais aussi provenant d'autres pays africains, notamment le Nigéria) ; Chinois (au moins 2000 personnes, pratiquant presque toutes le mandarin et éventuellement d'autres variétés de chinois); Européens (lusophones, francophones, anglophones, etc.). Le portugais, langue officielle du Cap-Vert, est également pratiqué en tant que langue maternelle (souvent en complémentarité avec la LCV) par une minorité de Capverdiens (probablement moins de $5 \%$ ).

5. La dichotomie «acrolectal» vs "basilectal» se fonde sur Bickerton (1975) où l'auteur considère néanmoins que la variété acrolectale désigne la langue lexificatrice elle-même (l'anglais dans la situation étudiée par Bickerton) et non une variété de la langue créole influencée par la langue lexificatrice, comme nous le faisons ici.

6. C'est sur Santiago que se trouve Praia, la capitale du Cap-Vert, qui regroupe à elle seule près d'un tiers de la population du pays.

7. C'est aussi au moyen du logiciel ELAN et en utilisant les annotations produites qu'ont été générées les requêtes débouchant sur les résultats quantitatifs présentés et discutés dans la troisième partie ("Analyse du corpus ») de cette étude.

8. On notera que, malgré leur proximité formelle, la marque santiagaise '-[de] de passif passé (morphème syncrétique codant à la fois une voix et un temps pour un verbe fini) ne provient pas diachroniquement de la marque de participe passé féminin du portugais (trouvée sous des formes proches presque partout en ibéro-roman) en '-[de] (morphème syncrétique codant à la fois un mode et un genre pour un verbe non fini), que l'on trouve par exemple dans PT cantada [kẽ'tade] 'chantée'.

9. L'expression du complément d'agent est de toute façon nettement minoritaire dans les langues qui y ont régulièrement recours. Ainsi, en français parlé, les énoncés passifs achevés (avec complément d'agent exprimé) représentent moins de $10 \%$ des énoncés passifs observés en corpus (Hamma, 2017, p. 3 ; Hamma et coll., 2017, p. 3 ; Hamma, 2020, p. 3).

10. Il existe toutefois des cas exceptionnels d'expression de l'agent, attestés par ailleurs: cf. Mendes et coll. (2002, p. 323-324, 'por'); Vieira Semedo (en préparation).

11. Ceci va dans le sens d'observations générales faites par les typologues (Givón, 2001, p. 39).

12. Dans le cadre de nos recherches sur la phrase complexe capverdienne, nous avons fait le choix de considérer comme des subordonnées complétives les énoncés en discours direct introduits par un verbe déclaratif. Pour plus de détails sur ce choix, cf. Vieira Semedo (en préparation).

13. Le rapport est presque de 1 à 6 si on retient la première décimale : 1,6 contre 9,5 .

14. Cette étiquette désigne des propositions ayant une structure de subordonnées mais se comportant syntaxiquement comme des propositions indépendantes. Pour plus de détails, cf. Vieira Semedo (en préparation).

15. Seuls 64 des 65 passifs ont été pris en compte ici. La forme passive restante apparait dans une proposition indépendante, un type peu attesté dans le corpus et qui n'a pas fait l'objet d'un traitement statistique propre à ce stade de nos recherches.

16. Ici, le nombre total de propositions (3 852) est inférieur au nombre de verbes fléchis (4 057, cf. 1.2). Cette différence est essentiellement due à l'absence de prise en compte des propositions indépendantes (cf. note précédente).

17. En capverdien, le passé morphologique permet de situer explicitement l'action verbale par rapport à un repère temporel situé avant le repère temporel du présent de l'énonciation. Une 
action décrite avec un présent morphologique peut notamment avoir un ancrage temporel passé : c'est en particulier souvent le cas pour les verbes dits « faibles » quand ils sont employés à l'aspect accompli (marque zéro). Un verbe faible accompli dénotant une action complètement effectuée au moment de l'énonciation se situe dans la sphère du présent morphologique, mais dénote un évènement objectivement situé dans le passé (Quint, 2000a, p. 229-240 ; 2010, p. 37, $246-249 ; 2012$, p. 162) : c'est ce qui explique que, même dans un contexte narratif comme celui des contes traditionnels, le présent morphologique puisse dominer en capverdien.

18. En synchronie, le suffixe du passif passé '-[de] n'est pas plus complexe que le suffixe du passif présent '-[du]. Cependant, si l'on tient compte du fait que, d'un point de vue diachronique, '-[de] est le résultat de la fusion de deux suffixes (passif + passé, cf. 2.1 ci-dessus), on peut admettre que l'usage de '-[de] est morphologiquement plus marqué que celui de '-[du].

19. Pour les interactions entre aspect et passif d'un point de vue typologique, cf. Keenan et Dryer (2007, p. 340-341, 360).

20. Ici le total des verbes considérés dans le corpus est réduit, car nous n'avons pas pris en compte les formes qui ne sont pas combinables avec les marques d'aspect, notamment les impératifs et les formes présente é et passée éra de la copule.

21. Cette apparente neutralité de la négation par rapport à la voix n'est pas un universel linguistique : certaines variétés de peul disposent ainsi, au moins sur le plan morphologique, de négations verbales distinctes pour chacune de leurs voix (active, moyenne et passive); cf. Labatut (1976, p. 452-453, apud Creissels, 1991, p. 441).

22. La fréquence des formes verbales négatives dans l'ensemble du corpus est probablement légèrement surévaluée, car certains adjectifs (non comptés comme verbes) se combinent avec la négation $k a$ et se comportent alors comme noyaux prédicatifs (Quint, 2000a, p. 265-266). Ces cas sont rares et le biais peut être considéré comme négligeable.

23. Pour des raisons de place, les traductions de la glose ci-dessus sont approximatives : djangáda désigne le brancard mortuaire dans lequel on exposait autrefois le mort avant de l'enterrer tandis que karís se réfère à la canne de Provence, plante dont l'aspect est assez proche de celui du roseau.

\section{RÉSUMÉS}

Le capverdien santiagais connait une voix passive synthétique, utilisée principalement pour promouvoir un objet au rang de sujet ou dans des constructions impersonnelles. Le présent article fait le point sur le passif santiagais à partir de l'examen d'un corpus annoté de textes oraux. Dans une première partie, nous présentons la langue capverdienne et la méthodologie utilisée pour collecter et traiter les données. Puis, dans une deuxième partie, nous fournissons des éléments sur la morphologie du passif verbal en capverdien santiagais. La troisième partie, en se fondant sur des données quantitatives et qualitatives issues du corpus, traite de la fréquence des formes passives du capverdien, de leur interaction avec les marques de temps, d'aspect et de polarité, des actants impliqués dans les constructions passives, ainsi que de la valence et du profil lexical des verbes attestés au passif. L'article conclut sur l'apport des données issues d'un corpus à l'étude du passif en capverdien et sur les possibilités d'améliorer et d'amplifier les résultats obtenus dans le futur. 
Santiaguense Capeverdean has a synthetic passive voice, which is mainly used either to promote an object to the status of subject or in impersonal constructions. This study takes stock of Santiaguense passive based on the scrutiny of an annotated corpus of spoken texts. In section one, we give a short account of the Capeverdean language and the methodology used to collect and process the data. In section two, we provide detailed information about passive morphology in Santiaguense Capeverdean. Then, in section three, we draw on both quantitative and qualitative data from our corpus to check the frequency of Capeverdean passive forms, the way they interact with tense, aspect and polarity, the arguments involved in passive constructions, as well as the valency and lexical peculiarities of the verbs that exhibit passive morphology. The conclusion emphasizes the interest of such a corpus-based approach for a fuller understanding of the passive voice in Capeverdean and suggests means to better and amplify the results achieved in this study for future research.

A variedade de Santiago do crioulo cabo-verdiano tem uma voz passiva sintética, utilizada principalmente para promover um objeto à categoria de sujeito ou para construções impessoais. Este artigo faz um estudo sobre a voz passiva em santiaguense a partir da análise de um corpus de textos orais. Na primeira parte, apresentamos a língua cabo-verdiana e a metodologia utilizada para a recolha e o tratamento dos dados. Na segunda parte, fornecemos elementos sobre a morfologia dos verbos passivos no santiaguense. Na terceira parte, baseando-nos nos dados quantitativos e qualitativos do corpus, tratamos da frequência das formas passivas em caboverdiano, da sua interação com as marcas de tempo, aspecto e polaridade e com os actantes envolvidos nas construções passivas, bem como a valência e o perfil lexical dos verbos atestados na voz passiva. $O$ artigo traz conclusões sobre o contributo de dados de um corpus para o estudo do passivo cabo-verdiano e sobre as possibilidades de melhorar e ampliar os resultados obtidos no futuro.

\section{INDEX}

Keywords : Capeverdean, corpus studies, passive voice, Portuguese, Romance, Upper Guinea Creoles (UGC)

Mots-clés : capverdien, créoles à base portugaise de l'Afrique de l'Ouest (CPAO), Corpus, langues romanes, passif, portugais

\section{AUTEURS}

NICOLAS QUINT

LLACAN - UMR 8135 (CNRS / INALCO / USPC)

ELIANE VIEIRA SEMEDO

LLACAN - UMR 8135 (CNRS / INALCO / USPC) 